\title{
DISFUNCIÓN ERÉCTIL DE ORIGEN HORMONAL
}

José María Martínez Jabaloyas.

Servicio de Urología. Hospital Clínico Universitario. Valencia. España.

Resumen.- El adecuado funcionamiento del mecanismo de la erección depende de la correcta interrelación de factores psicológicos, vasculares, neurológicos y hormonales. Las enfermedades endocrinas afectan a la función sexual y la disfunción sexual puede ser uno de los síntomas de alguna alteración hormonal.

La diabetes mellitus es la alteración endocrina que con más frecuencia causa disfunción eréctil debido a las frecuentes complicaciones vasculares y neurológicas que conlleva. Es importante la determinación de glucosa en la evaluación inicial del varón con disfunción eréctil, asi como intentar un adecuado control de los niveles de

\section{CORRESPONDENCIA}

José Mํa Martínez Jabaloyas

Servicio de Urología

Hospital Clínico Universitario

Avda Blasco Ibáñez 17

46010 Valencia (España)

jabaloyas@telefonica.net glucemia para evitar el empeoramiento. La disfunción eréctil del diabético es multifactorial, de mayor severidad y responde peor al tratamiento oral.

La hiperprolactinemia es causante de alteraciones en la esfera sexual, ya que provoca descenso de la testosterona. En estos casos, la sintomatología sexual se trata corrigiendo los niveles de prolactina. La determinación rutinaria de la prolactina no está clara y parece que debe determinarse cuando las cifras de testosterona están disminuidas.

Las alteraciones de la hormona tiroidea (tanto el hiper como el hipotiroidismo) se asocian a disfunción eréctil, que en la mitad de los casos remitirá con la normalización de la hormona tiroidea. No está claro el papel que juegan las hormonas suprarrenales en la función eréctil y su determinación rutinaria no se contempla en la evaluación diagnóstica de la disfunción eréctil. Tampoco se conoce bien el papel del estradiol en la regulación del mecanismo de la erección, aunque se sabe que cifras elevadas de este pueden provocar disfunción eréctil. Dentro de las alteraciones endocrino-metabólicas reseñar las dislipemias, con la hipercolesterolemia como factor de riesgo importante de disfunción eréctil y aunque su corrección puede prevenir el deterioro del sistema vascular, no está claro el papel de las estatinas en la disfunción eréctil.

Palabras clave: Disfunción eréctil. Hormona. Diabetes mellitus. Prolactina. Hormona tiroidea. Estradiol.

Summary.- The proper function of erection mechanisms depend on correct interrelationship between psychological, vascular, neurological and hormonal factors. Endocrine diseases affect sexual function, and sexual dysfunction may be one of the symptoms of some hormonal anomalies. 
Diabetes mellitus is the endocrine disease most frequenthy causing erectile dysfunction due to the frequent vascular and neurological complications associated. It is important to determine blood glucose in the initial evaluation of a male with erectile dysfunction, as well as to try an adequate control of blood glucose levels to avoid worsening. Diabetic male erectile dysfunction is multifactorial, more severe and has worse response to oral treatment.

Hyperprolactinemia causes disorders of the sexual sphere because it produces a descent of testosterone. In these cases, sexual symptoms are treated by correcting the levels of prolactin. Routine determination of prolactin is not clear and it seems it should be determined when testosterone levels are diminished.

Thyroid hormone disorders (both hyper and hypotyroidism) are associated with erectile dysfunction, which will subside in half the patients with thyroid hormone normalization. The role of adrenal hormones in erectile function is not clear and their routine determination is not considered in the diagnostic evaluation of erectile dysfunction. The role of estradiol in the regulation of the erection mechanism is not well known either, although it is known that high levels may cause erectile dysfunction. Among endocrine-metabolic disorders we point out dyslipemias, with hypercholesterolemia as an important risk factor for erectile dysfunction and, though its correction may prevent vascular system deterioration, the role of statins in erectile dysfunction is not clear.

Keywords: Erectile dysfunction. Hormone. Diabetes Mellitus. Prolactin. Thyroid hormone. Estradiol.

\section{INTRODUCCIÓN}

Una función sexual adecuada depende de la correcta interrelación de factores psicológicos, emocionales, neurológicos, vasculares y endocrinos. Si bien la principal causa de disfunción eréctil es la alteración del sistema vascular, no hay que olvidar que determinados trastornos endocrinos pueden originar o agravar un cuadro de disfunción eréctil. Los trastornos endocrinos aumentan con la edad y la población está envejeciendo con un aumento de la prevalencia de la obesidad y la diabetes, por lo que estos trastornos van a contribuir de forma creciente en el desarrollo de cuadros de disfunción eréctil. Se realiza una revisión de los principales trastornos endocrinos que han mostrado algún tipo de asociación con la disfunción eréctil.

\section{Diabetes Mellitus y disfunción eréctil}

Podemos considerar la diabetes mellitus como la enfermedad endocrina más importante que da lugar a un cuadro de disfunción eréctil. Sin embargo, aunque etiológicamente sea la causa primaria, la alteración del metabolismo glucídico no es el causante directo de la disfunción eréctil, sino que es el desencadenante de alteraciones vasculares, neurológicas y endocrinas que serán las que en última instancia perturbarán el mecanismo de la erección.

La diabetes Mellitus tipo I y II se caracterizan por tener cifras elevadas de glucemia, aunque ambas entidades difieren en importantes factores como la insulina, el índice de masa corporal, así como el perfil lipídico y las citoquinas. Aunque algunas características de la disfunción eréctil en la diabetes Mellitus I y ll son similares, cada una tiene particularidades diferentes ya que los mecanismos que ocasionan la alteración de la erección no son idénticos. Por ello, los estudios experimentales y epidemiológicos deberían diferenciar entre ambas entidades (1). Es importante determinar la glucosa como prueba complementaria en el diagnóstico de la disfunción eréctil, ya que hay estudios que refieren hasta un $12 \%$ de diabéticos no conocidos entre los pacientes que consultan por esta patología (2).

La prevalencia de la disfunción eréctil entre diabéticos es muy variable en base a los criterios establecidos para realizar el diagnóstico de disfunción eréctil y la población incluida en el estudio. Así, según las series puede variar del 10 al $90 \%$ (3). Los estudios no suelen distinguir entre tipo I y tipo II, por lo que es difícil establecer si es más frecuente en un tipo $u$ otro de diabetes. Por otra parte se ha reportado que la disfunción eréctil es 3 veces más frecuente en diabéticos y que aparece entre 10 y 15 años antes en los varones diabéticos (3).

Se han reportado como factores de riesgo para la disfunción eréctil la edad y el tiempo de duración de la diabetes, así como el mal control de la glucemia y el sedentarismo $(4,5)$. En el diabético es frecuente que coexistan otros factores de riesgo para la disfunción eréctil como la hipertensión arterial (así como algunos fármacos para su tratamiento), la dislipemia y la obesidad (6).

Los mecanismos patogénicos que provocan la disfunción eréctil son principalmente orgánicos, aunque no hay que menospreciar la afectación psicológica que conlleva una enfermedad crónica con gran número de complicaciones como la diabetes. En este sentido se ha descrito un mayor riesgo de depresión entre los diabéticos (7). Entre los factores 
orgánicos cabe destacar las alteraciones vasculares que comienzan con la disfunción endotelial, apareciendo en los diabéticos una mayor contractibilidad del tejido cavernoso y una frecuente disfunción corporovenooclusiva (8). La neuropatía es una complicación frecuente en la diabetes Mellitus, de forma que se han descrito casos en los que la disfunción de los nervios peneanos antecede a la de otros nervios periféricos (9). Por otra parte, la frecuencia de hipogonadismo es alta entre los varones con diabetes Mellitus tipo II e incluso se ha asociado los niveles de testosterona al control metabólico (10), por lo que es importante la determinación de testosterona en la evaluación diagnóstica de este grupo de pacientes.

Aunque el manejo de la disfunción eréctil en los diabéticos no difiere respecto al resto de los varones con esta patología, hay que tener en cuenta que el cuadro suele ser más severo y se asocia a peor calidad de vida (1 1). El primer escalón terapéutico será el mismo, los inhibidores de la fosfodiesterasa 5, aunque la respuesta a este tipo de tratamiento va a ser más baja, con mayor tasa de fracasos terapéuticos
(12), por lo que deberemos recurrir con más frecuencia a las otras alternativas, como son las inyecciones intracavernosas y los dispositivos de vacío, sin olvidar que el tratamiento del hipogonadismo (muy frecuente en estos pacientes) puede favorecer la acción de los inhibidores de la fosfodiesterasa, con lo que la normalización de las cifras de testosterona pueden rescatar a un importante número de pacientes para el tratamiento oral (13). Cuando estos tratamientos fallan o no son bien tolerados, la prótesis peneana es una buena alternativa, con tasas de satisfacción muy elevadas (14). Es conveniente recordar que el riesgo de infección de la prótesis va a ser mayor en la población diabética (15).

\section{Deficiencia androgénica}

La testosterona juega un papel fundamental en la respuesta sexual masculina. El déficit de testosterona puede dar lugar a alteraciones en toda la esfera sexual, lo que será tratado en un capítulo de este monográfico.

\section{TABLA I. FÁRMACOS QUE PUEDEN OCASIONAR HIPERPROLACTINEMIA (26).}

\section{- Antipsicóticos y otros bloqueadores de receptores dopaminérgicos}

- Fenotiacinas (chlopromazine, mesoridazine, thioridazine, fluphenazine, perphenazine, trifluoperazine)

- Butirofenonas (haloperidol, pimozide, fluspirilene, penfluridol, risperidone)

- Benzamidas (sulpiride, amisulpride, levosulpiride, cisapride, metoclopramida)

- Tioxantinas (clorprotixen, tiotixeno)

- Inhibidores de la síntesis de dopamina: $\alpha$-Metildopa

- Fármacos que producen depleción de catecolaminas: Reserpina

\section{- Antidepresivos}

- Inhibidores de la recaptación de serotonina (citalopram, paroxetina, sertralina, fluoxetina, fluvoxamina, escitalopram)

- Inhibidores de la recaptación de Serotonina-norepinefrina/

Antidepresivos atípicos (venlafaxina, trazodona, mirtazapina, bupropion)

- Tricilicos (clorimipramina, amitriptilina)

\section{- Opiaceos}

- H2 antagonistas: Cimetidina, Ranitidina

- Bloqueantes de los canales del calico: Verapamilo

- Hormonas: Estrógenos, Antiandrógenos

- Anticonvulsivantes: Fenitoina 


\section{Hiperprolactinemia}

La prolactina es una hormona polipeptídica secretada por la pituitaria. Aunque fue descubierta en 1933 (16), aún no se conoce su función en el hombre. Sin embargo sí que es bien conocida que la elevación de la prolactina puede causar alteraciones en el comportamiento sexual y reproductivo $(17,18)$.

La presencia de cifras moderadamente elevadas $(>20 \mathrm{ng} / \mathrm{ml}$ ) en varones con disfunción eréctil varía del 2 al 13\% $(17,19)$. Las hiperprolactinemias severas $(>35 \mathrm{ng} / \mathrm{ml}$ ) son más raras y afectan a menos del $1 \%(17,18)$. Las elevaciones de la prolactina pueden ser transitorias (por la misma secreción pulsátil de la prolactina) y pueden estar condicionadas por situaciones de estrés como puede ser la misma extracción sanguínea, por lo que es conveniente la confirmación diagnóstica cuando en un análisis se detectan cifras altas de prolactina. Las hiperprolactinemias leves no alternan la función sexual siendo necesario que la hiperprolactinemia sea severa (18), ya que el límite establecido en que se considera que la hiperprolactinemia puede afectar a la función sexual es de $35 \mathrm{ng} / \mathrm{ml}$ (17). Se ha descrito una asociación entre hiperprolactinemia y desórdenes mentales, aunque este extremo, una vez descartados los psicofármacos, no se ha corroborado (18).

El síntoma más importante de hiperprolactinemia es la disfunción eréctil (aparece en el $88 \%$ de los casos), típicamente asociado a pérdida del deseo sexual, aunque no necesariamente. Puede aparecer retraso o pérdida del orgasmo, pero siempre asociado a disfunción eréctil, y más raramente alteraciones en la eyaculación. Además de las alteraciones sexuales y con menos frecuencia, puede aparecer disminución del vello corporal (40\%) y ginecomastia (13\%) $(17,19)$. La galactorrea en varones es poco frecuente (20). Curiosamente se ha reportado una mayor prevalencia de eyaculación precoz en varones con hiperprolactinemia leve (19) sin que la causa de esta asociación esté clara.

La causa por la que la hiperprolactinemia produce disfunción eréctil no está totalmente aclarada. Se ha atribuido a la disminución de las cifras de testosterona sérica por alteración en la secreción de LH (18), por ello y por la baja frecuencia de hiperprolactinemias significativas en los varones con disfunción eréctil, se recomienda la determinación de la prolactina únicamente cuando las cifras de testosterona estén bajas (21-23). También la pérdida de deseo sexual se ha asociado a las cifras bajas de testosterona (18). Sin embargo, como motivo de controversia, se han descrito casos de disfunción eréctil por hiperprolactinemia con cifras de testosterona normales (24). Así la disfunción eréctil no solo se atribuye al descenso de la testosterona sino también por afectación directa del pene por la hiperprolactinemia (23).

El diagnóstico de hiperprolactinemia precisa de una confirmación del resultado de laboratorio, repitiendo el análisis o realizando una determinación de pulsos de prolactina, ya que el estrés ocasionado por la misma punción venosa puede ser causa de elevación. Una de las causas de falsos positivos es la macroprolactinemia, que se trata de un complejo de prolactina unida a un anticuerpo $\lg G$ con poca bioactividad pero con mayor vida media que la prolactina habitual, lo que condiciona su menor aclaramiento y la consiguiente acumulación de altas concentraciones séricas. Se sospechará ante una hiperprolactinemia de etiología no definida, con escasa repercusión clínica y falta de respuesta al tratamiento médico con agonistas dopaminérgicos. La medición de un valor de prolactina menor al $50-70 \%$ de la previa tras precipitación del suero con polietilenglicol es diagnóstica (25).

Las causas de hiperprolactinemia son diversas como herpes zóster, insuficiencia renal crónica, cirrosis, lesiones hipotalámicas (síndrome de silla turca vacía o craneofaringiomas) diversas medicaciones (Tabla I) y los adenomas pituitarios. Entre las medicaciones las que con más frecuencia causan hiperprolactinemia son los antipsicóticos (26), así aunque la causa más importante de hiperprolactinemia es el adenoma hipofisario secretor de prolactina, la causa más frecuente son los fármacos. Los adenomas se clasifican en microadenomas $(<10 \mathrm{~mm}$ ) y macroadenomas $(>10 \mathrm{~mm})$. El adenoma de células lactotropas o prolactinoma es el tumor hipofisario secretor más frecuente y representa el $60 \%$ de los adenomas funcionantes. El $90 \%$ de los prolactinomas son microadenomas intraselares. En los varones son más frecuentes los macroadenomas $(60 \%)$ ya sea porque la sintomatología es escasa, porque tardan en consultar o por una mayor tasa de crecimiento que en la mujer (25).

Cuando se confirma la hiperprolactinemia la principal exploración complementaria a realizar, una vez se descarta la causa farmacológica, es la Resonancia Nuclear Magnética, con el fin de descartar la presencia de adenomas hipofisarios.

Una vez establecida la causa instauraremos el tratamiento. En el caso de que la causa sea farmacológica se valorará su retirada o sustitución. El tratamiento de elección para los microadenomas y macroadenomas son los agonistas dopaminérgicos, independientemente del tamaño. Los agonistas dopaminérgicos utilizados son la bromocriptina, la cabergolina y el quinagolide $(20,25)$. 
La decisión de la cirugía se basa en el tamaño tumoral y la falta de respuesta del tratamiento médico y sus indicaciones son: macroadenomas invasivos que no reducen el tamaño y pacientes con microadenomas que no toleran los agonistas dopaminérgicos o no son efectivos. La radioterapia externa, radiocirugía o la cirugía esteroatáxica no es efectiva como tratamiento primario, generalmente se utiliza como adyuvante en tumores invasivos (20).

La normalización de las cifras de prolactina suele ir acompañada de una normalización de las cifras de testosterona, mientras que el tratamiento con suplementos de testosterona, mientras no se corrija la hiperprolactinemia, no servirá para mejorar la disfunción eréctil $(17,27)$. En el caso de que al normalizar las cifras de prolactina no se normalicen las cifras de testosterona deberán pautarse suplementos de testosterona.

\section{Alteración de la hormona tiroidea}

La mayoría de pacientes con alteración de la función tiroidea presentan una anomalía en la función sexual, que puede ser a nivel del deseo, de la erección o de la eyaculación (28), resolviéndose la mitad de los casos cuando se corrige la función tiroidea.

En un estudio llevado a cabo por Krassas et al se describe que existe disfunción eréctil casi en el $80 \%$ de un grupo de varones con alteración de la función tiroidea, bien por hipertiroidismo o por hipotiroidismo, frente a un $34 \%$ de un grupo control con las mismas características (29). Únicamente con la normalización de la función tiroidea se restauró la normalidad de la función eréctil en más de la mitad de los casos, quedando la frecuencia de disfunción eréctil similar al grupo control. Aunque estos autores abogan por un screening de enfermedad tiroidea en los pacientes con disfunción eréctil, y hay estudios que justifican su determinación (30), no está claro que su determinación rutinaria sea rentable, salvo que asocien otro tipo de sintomatología acompañante que así lo aconseje.

Por otra parte los varones con hipotiroidismo tienen menores concentraciones de testosterona libre y de SHBG que los controles, lo que puede contribuir al empeoramiento de la función sexual. El tratamiento del hipotiroidismo restaura los niveles hormonales a la normalidad (31).

En cuanto a la relación de las hormonas tiroideas con la eyaculación, se ha visto que un considerable número de pacientes con hipertiroidismo tienen eyaculación precoz observando un incremento considerable del tiempo de latencia eyaculatorio intravaginal cuando se corrige el hipertiroidismo y normalizándose la función eyaculatoria en más de la mitad de los casos (32).

\section{Alteraciones adrenales}

La incidencia de disfunción sexual en pacientes con insuficiencia adrenal, primaria y secundaria, es desconocida. La insuficiencia adrenal primaria se asocia con descenso de la producción de glucocorticoides, mineralcorticoides y de los andrógenos suprarrenales dehidroepiandroesterona, sulfato de dehidroepiandrosterona y androstendiona, mientras que en la insuficiencia adrenal secundaria no existe alteración de los mineralcorticoides. Aunque las enfermedades de las glándulas suprarrenales pueden ir acompañadas de disfunción eréctil, no existe ningún dato que indique que debamos descartar estas enfermedades en un varón con disfunción eréctil.

El papel de los andrógenos suprarrenales en la función eréctil no está bien establecido. Al igual que con la testosterona los niveles de dehidroepiandrosterona descienden con la edad $(33,34)$ y hay estudios que encuentran una relación entre los niveles bajos de dehidroepiandrosterona y la disfunción eréctil $(33,35)$. Un estudio piloto mostró mejora de la disfunción eréctil con el tratamiento con suplementos de dehidroepiandrosterona (36), pero con los datos que tenemos hasta el momento no existe justificación para solicitar de forma rutinaria la dehidroepiandrosterona o su forma sulfato, ni para instaurar tratamientos con suplementos de dehidroepiandrosterona para la disfunción eréctil.

La androstendiona es otro andrógeno producido en las glándulas suprarrenales (también se produce en los testículos) que puede ser convertido a testosterona. Se ha reportado que el tratamiento con suplementos de androstendiona podría favorecer el desarrollo y la fuerza muscular, reducir la grasa y mejorar la libido y la función eréctil, así como enlentecer la aparición de síntomas relacionados con el envejecimiento (37). Sin embargo los datos son poco consistentes para recomendar su uso.

\section{Alteraciones del estradiol}

El estradiol es la hormona femenina por excelencia y aunque no está claro el papel que juega en la función sexual masculina, un exceso de estradiol va a producir signos de feminización y alteraciones en la función eréctil. Así, los pacientes con enferme- 
dades hepáticas crónicas presentan con frecuencia descenso del deseo sexual y disfunción eréctil, así como la aparición de características sexuales femeninas como ginecomastia y redistribución del vello. Esto es atribuido al incremento de la aromatización de los andrógenos a estrógenos y disminución del metabolismo de los estrógenos. Altos niveles de estrógenos inhiben la producción de LH con la consiguiente reducción de la testosterona. Además se asocian mayores niveles de SHBG lo que produce la disminución de los niveles de testosterona libre $(38,39)$.

Alteraciones en el balance testosterona-estradiol ha mostrado influir en la función eréctil (40) y aunque hay algún estudio clínico que indique que el ratio testosterona:estradiol puede tener importancia en la evaluación clínica de la disfunción eréctil, es un aspecto muy poco contrastado (41).

\section{Alteración del metabolismo lipídico y disfunción eréctil}

Aunque la alteración lipídica no es una alteración hormonal, entraría dentro del grupo de trastorno endocrino-metabólico. La dislipemia se ha considerado como un factor de riesgo para la disfunción eréctil. Además existe una alta prevalencia de pacientes con trastorno del metabolismo lipídico sin diagnosticar y que son detectados cuando consultan por un cuadro de disfunción eréctil (42). Estudios experimentales mostraron que la causa era el deterioro de la relajación endotelio-dependiente del músculo liso del cuerpo cavernoso como consecuencia de la hipercolesterolemia (43). La causa fundamental sería la disfunción endotelial que la alteración del metabolismo lipídico, fundamentalmente el del colesterol, provoca en el sistema vascular. Aunque está claro que la hipercolesterolemia es un factor de riesgo para la disfunción eréctil, la relación con la hipertrigliceridemia pura está poco definida aunque un estudio piloto reciente indica que puede existir cierta relación entre elevación de triglicéridos y disfunción eréctil (44).

Se ha observado que el tratamiento con estatinas produce una mejoría en la función endotelial, con lo que además del efecto preventivo de deterioro del sistema cardiovascular y por tanto de la función eréctil que supone el rebajar las cifras de colesterol, presupone que el tratamiento con estatinas mejorará la función eréctil y así lo muestra algún estudio (aunque metodológicamente limitado) (45). Sin embargo otro estudio reciente relaciona el uso de fármacos hipolipemiantes con un empeoramiento de la función eréctil (46), por lo que no está claro el papel que juegan este tipo de fármacos en la función sexual de los pacientes con dislipemia.

\section{BIBLIOGRAFÍA y LECTURAS}

\section{RECOMENDADAS ( ${ }^{*}$ lectura de interés $y^{* *}$ lectura fundamental)}

*1. Chitaley K. Type 1 and type 2 diabetic-erectile dysfunction: Same diagnosis (ICD-9), different disease? J Sex Med 2009;6(suppl3):262-268.

2. Lewis RW. Epidemiology of erectile dysfunction. Urol Clin North Am 2001;28:209-16.

**3. Malavige LS, Levy JC. Erectile Dysfunction in Diabetes Mellitus. J Sex Med. 2009; 6:1232-47.

4. Zheng H, Fan W, Li G, Tam T. Predictors for erectile dysfunction among diabetics. Diabetes Res Clin Pract 2006;71:313-9;

*5. Romeo JH, Seftel AD, Madhun ZT, Aron DC. Sexual function in men with diabetes type 2: Association with glycemic control. J Urol 2000;163: 788-91.

6. El-Sakka AI, Tayeb KA. Erectile dysfunction risk factors in noninsulin dependent diabetic Saudi patients. J Urol 2003;169:1043-7.

7. Anderson RJ, Freedland KE, Clouse RE, Lustman PJ. The prevalence of comorbid depression in adults with diabetes: A meta-analysis. Diabetes Care 2001;24:1069-78.

**8. Hidalgo-Tamola J, and Chitaley K. Type 2 diabetes mellitus and erectile dysfunction. J Sex Med 2009;6:916-926.

9. Bleustein CB, Arezzo JC, Eckholdt H, Melman A. The neuropathy of erectile dysfunction. Int J Impot Res 2002;14:433-9.

*10. El-Sakka AI, Sayed HM, Tayeb KA. Androgen pattern in patients with type 2 diabetes-associated erectile dysfunction: impact of metabolic control. Urology. 2009;74:552-9.

11. Penson DF, Latini DM, Lubeck DP, Wallace KL, Henning JM, Lue TF. Do impotent men with diabetes have more severe erectile dysfunction and worse quality of life than the general population of impotent patients? Results from the Exploratory Comprehensive Evaluation of Erectile Dysfunction (ExCEED) database. Diabetes Care 2003;26: 1093-9.

*12. Goldstein I, Lue TF, Padma-Nathan H, Rosen RC, Steers WD, Wicker PA. Oral sildenafil in the treatment of erectile dysfunction. Sildenafil Study Group. N Engl J Med 1998;338:1397-404.

*13. Shabsigh R, Kaufman JM, Steidle C, Padma-Nathan $\mathrm{H}$. Randomized study of testosterone gel as adjunctive therapy to sildenafil in hypogonadal men with erectile dysfunction who do not respond to sildenafil alone. J Urol 2004;172:658-63

14. Rajpurkar A, Dhabuwala CB. Comparison of satisfaction rates and erectile function in patients treated with sildenafil, intracavernous prostaglandin E1 and penile implant surgery for erectile dysfunction in urology practice. J Urol. 2003;170:159-63

15. Wilson SK, Carson CC, Cleves MA, Delk JR 2nd. Quantifying risk of penile prosthesis infection with 
elevated glycosylated hemoglobin. J Urol 1998; 159:1537-9.

16. Riddle O, Bates RW, Kydshorn SW. The preparation, identificaction and assay of prolactin -a hormone of anterior pituitary. Am J Physiol 1993; 105: 191-216.

*17. Buvat J. Hyperprolactinemia and sexual function in men: A short review. Int J Impot Res. 2003; 15:373-7

**18. Corona G. Mannucci E, Fisher AD, Lotti F, Ricca $\mathrm{V}$, Balercia G, et al. Effect of hyperprolactinemia in male patients consulting for sexual dysfunction. $\mathbf{J}$ Sex Med. 2007; 4: 1485-93.

19. El-Sakka AI, Hassoba HM, Sayed HM, Tayeb KA. Pattern of endocrinal changes in patients with sexual dysfunction. J Sex Med 2005;2:551-8.

20. Schlechte JA. Prolactinoma. N Engl J Med 2003; 349:21: 2935-2041.

21. Maatman TJ, Montague DK. Routine endocrine screening in impotence. Urology. 1986;27:499-502. Akpunonu BE, Mutgi AB, Federman DJ, York J,

22. Woldenberg LS. Routine prolactin measurement is not necessary in the initial evaluation of male impotence. J Gen Intern Med. 1994;9:336-338.

23. Zeitlin, SI, Rajfer J. Hyperprolactinemia and Erectile Dysfunction. Rev Urol. 2000;2(1):39-42.

24. Buvat $\mathbf{J}$ et al. Hyperprolactinemia and sexual function in men. Hormone Res 1985; 22: 196 - 203

25. Moreno B, Obiols G, Páramo C, Zugasti A. Guía clínica del manejo del prolactinoma y otros estados de hiperpolactinemia. Endorinol Nutr. 2005; 52: 9-17.

26. Molitch ME. Drugs and prolactin. Pituitary 2008;11:209-18

27. Ciccarelli A, Guerra E, De Rosa M, Milone F, Zarrilli S, Lombardi G, Colao A. PRL secreting adenomas in male patients. Pituitary 2005;8:39-42.

*28. Carani C, Isidori AM, Granata A, Carosa E, Maggi M, Lenzi A, Jannini EA. Multicenter study on the prevalence of sexual symptoms in male hypo- and hyperthyroid patients. J Clin Endocrinol Metab. 2005;90:6472-9.

29. Krassas GE, Tziomalos K, Papadopoulou F, Pontikides N, Perros P. Erectile dysfunction in patients with hyper- and hypothyroidism: how common and should we treat? J Clin Endocrinol Metab. 2008;93:1815-9.

*30. Bodie J, Lewis J, Schow D, Monga M. Laboratory evaluations of erectile dysfunction: an evidence based approach. J Urol. 2003;169:2262-4.

31. Donnelly $\mathrm{P}$, White $\mathrm{C}$. Testicular dysfunction in men with primary hypothyroidism; reversal of hypogonadotrophic hypogonadism with replacement thyroxine. Clin Endocrinol (Oxf). 2000;52:197-201.

*32. Cihan A, Demir O, Demir T, Aslan G, Comlekci A, Esen A. The relationship between premature ejaculation and hyperthyroidism. J Urol. 2009;181:1273-80.

33. Feldman HA, Goldstein I, Hatzichristou DG, Krane RJ, McKinlay JB. Impotence and its medical and psychosocial correlates: results of the Massachusetts
Male Aging Study. J Urol. 1994;151:54-61

34. Martínez Jabaloyas JM, Queipo Zaragoza A, Ferrandis Cortes C, Queipo Zaragoza JA, Gil Salom M, Chuan Nuez P. Cambios en las hormonas sexuales en varones mayores de 50 años. Prevalencia de niveles bajos de testosterona y factores de riesgo. Actas Urol Esp. 2008 ;32:603-10.

*35. Reiter WJ, Pycha A, Schuatzl G, et al. Serum dehidroepiandrosterona sulfate concentrations in men with erectile dysfunction. Urology. 2000; 55: 755-8.

36. Reiter WJ, Pycha A, Schatzl G, Pokorny A, Gruber DM, Huber JC, et al. Dehydroepiandrosterone in the treatment of erectile dysfunction: a prospective, double-blind, randomized, placebo-controlled study. Urology 1999;53:590-4.

37. Tamler R, Mechanick JI. Dietary supplements and nutraceuticals in the management of andrologic disorders. Endocrinol Metab Clin North Am. 2007;36:533-52.

38. Guechot J, Chazouilleres O, Loria A, Hannoun L, Balladur P, Parc R, Giboudeau J, Poupon R. Effect of liver transplantation on sex-hormone disorders in male patients with alcohol-induced or post-viral hepatitis advanced liver disease. J Hepatol 1994;20:426-30.

39. Madersbacher S, Ludvik G, Stulnig T, Grunberger $\mathrm{T}$, Maier U. The impact of liver transplantation on endocrine status in men. Clin Endocrinol (Oxf) 1996;44:461-6.

40. Adaikan PG, Srilatha B. Oestrogen-mediated hormonal imbalance precipitates erectile dysfunction. Int J Impot Res. 2003;15:38-43.

41. Srilatha B, Adaikan PG, Chong YS. Relevance of oestradiol-testosterone balance in erectile dysfunction patients' prognosis. Singapore Med J. 2007 ;48:114-8.

42. Smith NJ, Sak SC, Baldo O, Eardley I. The prevalence of newly diagnosed hyperlipidaemia in men with erectile dysfunction. BJU Int. 2007;100:357-61.

43. Azadzoi K, Saenz de Tejada I. Hypercholesterolemia impairs endothelium-dependent relaxation of rabbit corpus cavernosum smooth muscle. J Urol 1991;146:238.

44. Heruti R, Arbel Y, Steinvil A, Zarka S, Saar N, Kinori M, Brenner G, Justo D. Pure hypertriglyceridemia might be associated with erectile dysfunction: a pilot study. J Sex Med. 2008;5:1230-6.

45. Gokkaya SC, Ozden C, Levent Ozdal O, Hakan Koyuncu H, Guzel O, Memis A. Effect of correcting serum cholesterol levels on erectile function in patients with vasculogenic erectile dysfunction. Scand J Urol Nephrol. 2008;42:437-40.

46. Hall SA, Kupelian V, Rosen RC, Travison TG, Link CL, Miner MM, Ganz P, McKinlay JB.Is hyperlipidemia or its treatment associated with erectile dysfunction?: Results from the Boston Area Community Health (BACH) Survey. J Sex Med. 2009 May;6(5):1402-13. 